\title{
THE MAGNETIC STRUCTURING OF THE SOLAR CORONA
}

\author{
A. W. HOOD \\ University of St Andrews, UK.
}

\section{Introduction}

The magnetic structuring of the solar corona can be seen during a solar eclipse. The $X$-ray corona has been photographed during recent satelite missions and balloon flights and it is made up of a few main building blocks including (i) coronal loops and (ii) X-ray bright points. These appear to be connected with interacting loops and magnetic cancellation. Much cooler structures, called, quiescent prominences, are also visible in the corona and these are supported against gravity in a stable magnetic configuration.

\section{Coronal Loops}

$U V$ and $X$-ray images of coronal loops show that they are thin, curved and almost toroidal in shape. The angle the field line is twisted through in going from one end of the loop to the other, $\Phi$ is an important stability parameter. Coronal loops must be stable against ideal MHD disturbances since their lifetimes greatly exceed the local Alfvén timescale. An important stabilising feature, called line-tying, occurs because the coronal magnetic field lines penetrate the dense photosphere (about $10^{8}$ times denser than the corona). Including line-tying it has been shown that force-free loops become unstable if the average twist, $\langle\Phi\rangle$, exceeds $2.5 \pi$.

It is thought that the solar corona is heated by a mechanism involving the local magnetic field. It is likely this is time dependent and the dynamic and thermal response of the coronal plasma can be investigated by imposing a heating term of the form $H=H_{0}(1+\sin \omega t)$. The values of $H_{0}$ and $\omega$ necessary to keep a coronal loop hot can be obtained. There is a critical value of $\omega$ above which the loop remains hot and below which the plasma always cools down to chromospheric values. It is related to $1 / \tau_{\text {rad }}$, where $\tau_{\text {rad }}$ is the optically thin radiative timescale for the loop. 


\section{X-Ray Bright Points}

High resolution X-ray images of the corona reveal that X-ray bright points are small interacting loops that lie above regions in the photosphere where magnetic flux is cancelling. These observsations have led to a model of bright points by Priest, Parnell and Martin (Ap. J., 427,1994). Flux sources on the photospheric boundary are moved together and the field reconnects. The magnetic energy is converted into heat and the newly reconnected field lines give the shape of the bright points. There is good agreement between the theory and the observations. The model suggests that there are three phases of development, namely an preinteraction phase, an interaction phase and a cancellation phase during which photospheric reconnection annihilates some of the photospheric flux fragments and releases magnetic energy to heat the bright point.

\section{Prominences}

Quiescent prominences are divided into two main classes namely normal polarity prominences in which the magnetic field passes through the prominence in the same direction as the underlying photospheric field and inverse polarity prominences in which the field is in the opposite direction to that predicted from the photospheric field. Fiedler and Hood (Solar Phys.,146, 1993) have presented numerical models of normal polarity prominences of finite height and width in which the photospheric footpoint motions are imposed. Their results predict many prominence properties that are in agreement with observations. 


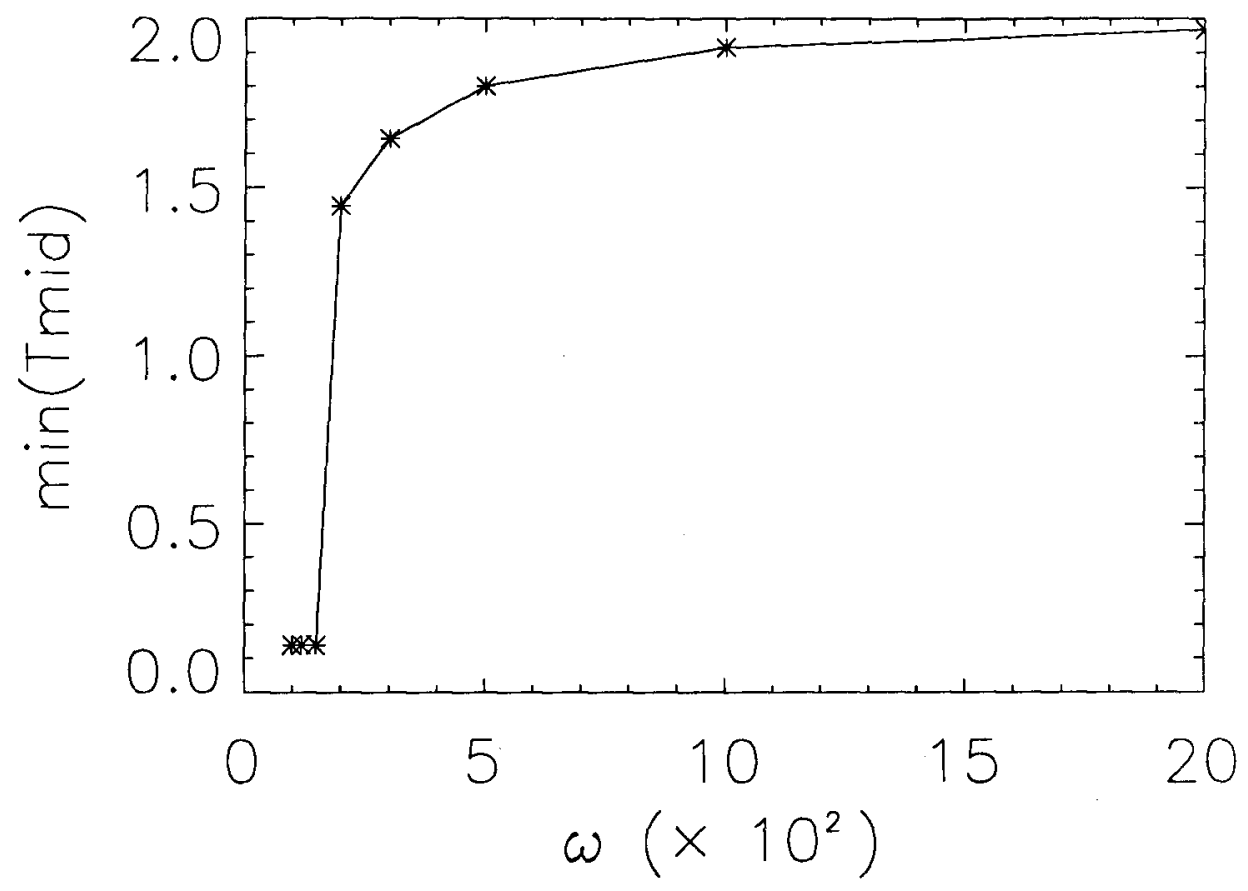

Figure 1. The variation of the minimum value of the temperature at the summit of a loop in units of $10^{6} \mathrm{~K}$, as a function of the heating frequency, $\omega$. 